\title{
Excited state spectroscopy in the lattice Gross-Neveu model
}

\author{
Julia Danzer*, Christof Gattringer \\ Karl-Franzens Universität Graz, Austria, Institut für Physik \\ E-mail: julia.danzer@edu.uni-graz.at, \\ christof.gattringereuni-graz.at
}

We present preliminary results of an excited state spectroscopy calculation in the 2-d lattice GrossNeveu model. We address the construction of suitable interpolators for the variational method and their overlap with excitations. We comment on the role of the eigenvectors as a tool for matching scattering states on lattices with different volumes.

The XXV International Symposium on Lattice Field Theory

July 30 - August 42007

Regensburg, Germany

${ }^{*}$ Speaker. 


\section{Introduction}

Lattice QCD is by now a mature field which produces reliable quantitative results from ab-initio calculations. With refined techniques the range of quantities that can be computed on the lattice is increasing continuously.

Observables that recently have started to see a lot of attention in the lattice community are masses of excited states of light hadrons (see e.g. [1]). Excitations, however, are quantities that are notoriously difficult on the lattice. The reason is that the energies $W_{n}$ of excited states appear only as sub-leading exponentials in the spectral decomposition of Euclidean 2-point functions,

$$
C_{i j}(t)=\left\langle O_{i}(t) O_{j}(0)^{\dagger}\right\rangle=\sum_{n}\left\langle 0\left|\widehat{O}_{i}\right| n\right\rangle\left\langle n\left|\widehat{O}_{j}^{\dagger}\right| 0\right\rangle e^{-t W_{n}} .
$$

Here $\widehat{O}_{i}, \widehat{O}_{j}$ are operators with the quantum numbers of the state one is interested in. The sum runs over all physical states $|n\rangle$ with these quantum numbers, and $W_{n}$ are the corresponding energies $\left(W_{1}<W_{2}<W_{3} \ldots\right)$. It is obvious, that the exponential decay of the 2-point function is dominated by the energy of the ground state $W_{1}$, while the energies of the excited states $W_{2}, W_{3} \ldots$ appear in subleading exponentials and thus their contribution to the 2-point function is exponentially suppressed relative to the ground state.

Although other proposals for the extraction of the excites state energies exist [2], the most commonly applied technique is the variational method [3,4]. Here one uses a large basis of interpolators $O_{i}, i=1,2, \ldots N$ and considers the generalized eigenvalue problem of the $N \times N$ correlation matrix $C(t)$,

$$
C(t) \vec{v}^{(n)}=\lambda(t)^{(n)} C\left(t_{0}\right) \vec{v}^{(n)} .
$$

The ordered eigenvalues, $\lambda^{(1)}>\lambda^{(2)}>\lambda^{(3)}>\ldots$, behave as [4]

$$
\lambda^{(n)}(t)=e^{-\left(t-t_{0}\right) W_{n}}\left[1+\mathscr{O}\left(e^{-\left(t-t_{0}\right) \Delta_{n}}\right)\right],
$$

where $W_{n}$ is the energy of the $n$-th state and $\Delta_{n}$ the distance of $W_{n}$ to the neighboring energy level. Effectively the ground and excited states are disentangled, and each energy appears in an individual eigenvalue. The variational method can only be as good as the basis of interpolators $O_{i}$ one uses and different construction principles were proposed [1].

Even with the variational method, the calculation of excited states is a challenging enterprise. Several non-trivial steps are involved: One has to construct a suitable basis of interpolators and select from those a subset which optimizes the quality of the signal, i.e., provides the cleanest effective mass plateaus. For an extrapolation to the infinite volume, continuum and chiral limits, the signals from several lattices with different size, coupling and mass have to be matched. Here it turns out that the eigenvectors $\vec{v}^{(n)}$ provide an important marker for identifying the states. Finally, in a fully dynamical simulation hadrons may decay. This leads to coexistence of bound- and scattering states which have similar energies $W_{n}$ and thus mix in the spectral sums (1.1). Because scattering states have relative momentum, they can be identified by a finite volume analysis, since the minimal possible momentum is inverse proportional to the spatial extent of the lattice [5].

Although the methods for solving the technical problems of an excited spectroscopy calculation are known, so far the typical analysis produced only the masses for one or two excitations and most papers are still in the quenched approximation, where hadrons cannot decay. 
There is a long standing tradition of studying problems of theoretical physics in low dimensional quantum field theories. This allows to test new concepts and techniques in an environment where often the situation is simpler and, when the lattice is used, large statistics is easy to generate (examples for such 2-d studies are [4], [6]). The experience gained in 2-d is then often an important guideline for the more demanding case of full QCD.

In this paper we follow this tradition and present first results of a lattice study of excited states in the 2-d Gross-Neveu model [7]. This model is particularly attractive for excited state spectroscopy, since in the infinite flavor limit, analytic results suggest that the masses of the excitations are integer multiples of the ground state mass. Our project aims at extracting with the variational method as many excitations as possible and to study their volume dependence to learn about scattering.

\section{Lattice simulation of the Gross-Neveu model}

\subsection{Setting of the calculation}

We use the Wilson action for discretizing the fermions on a 2-d lattice $\Lambda$. The total action is

$$
S[\bar{\psi}, \psi, \Phi]=\sum_{\vec{x}, \vec{y} \in \Lambda} \bar{\psi}(\vec{x}) D(\vec{x}, \vec{y}) \psi(\vec{y})+\frac{1}{2} \sum_{\vec{x} \in \Lambda} \Phi(\vec{x})^{2}
$$

The fields $\bar{\psi}, \psi$ are vectors of $N_{f}$ two-component spinors, one for each flavor, and we use matrix/vector notation for all indices. $\Phi$ denotes a one-component real scalar field, which generates the 4-fermi interaction of the model through a Hubbard-Stratonovich transformation. The Dirac matrix $D$ is diagonal in flavor space and reads

$$
D(\vec{x}, \vec{y})=[m+\sqrt{g} \Phi(n)] \delta_{\vec{x}, \vec{y}}-\sum_{\mu= \pm 1}^{ \pm 2} \frac{\mathbb{1} \mp \gamma_{\mu}}{2} \delta_{\vec{x}+\hat{\mu}, \vec{y}}
$$

The matrices $\gamma_{\mu}$ are given by the Pauli matrices, $\gamma_{1}=\sigma_{1}, \gamma_{2}=\sigma_{2}, \gamma_{5}=\sigma_{3}$.

The dynamical simulation of the model is done with standard Hybrid Monte Carlo methods and we work with $N_{f}=2,4$ and 6 flavors. Our $L_{1} \times L_{2}$ lattices have sizes $L_{1}=10 \ldots 24, L_{2}=$ 32 ...64 and we typically use four values of the coupling constant $g$ and several different masses $m$. Statistical errors are determined with the Jackknife-method. We find that statistics of a few hundred configurations per ensemble are sufficient for statistical errors in the one percent range. Throughout this paper we set the lattice constant equal to one and all results are in lattice units. 


\subsection{Interpolators}

For our analysis of excited states we use the following set of flavor singlet interpolators:

$$
\begin{aligned}
O_{1}(t) & =\bar{\psi}(x, t) \gamma_{5} \psi(x, t), \\
O_{2}(t) & =\bar{\psi}(x, t) \gamma_{1} \psi(x, t), \\
O_{3}(t) & =\frac{1}{2} \bar{\psi}(x, t) \gamma_{5}[\psi(x+n, t)+\psi(x-n, t)], \\
O_{4}(t) & =\frac{1}{2} \bar{\psi}(x, t) \gamma_{1}[\psi(x+n, t)+\psi(x-n, t)], \\
O_{5}(t) & =\frac{1}{4}[\bar{\psi}(x+m, t)+\bar{\psi}(x-m, t)] \gamma_{5}[\psi(x+n, t)+\psi(x-n, t)], \\
O_{6}(t) & =\frac{1}{4}[\bar{\psi}(x+m, t)+\bar{\psi}(x-m, t)] \gamma_{1}[\psi(x+n, t)+\psi(x-n, t)], \\
O_{8}(t) & =\frac{1}{4}[\bar{\psi}(x+m, t)+\bar{\psi}(x-m, t)][\psi(x+n, t)-\psi(x-n, t)], \\
O_{9}(t) & =\frac{1}{4}[\bar{\psi}(x+m, t)-\bar{\psi}(x-m, t)] \gamma_{5}[\psi(x+n, t)-\psi(x-n, t)], \\
O_{10}(t) & =\frac{1}{4}[\bar{\psi}(x+m, t)-\bar{\psi}(x-m, t)] \gamma_{1}[\psi(x+n, t)-\psi(x-n, t)] .
\end{aligned}
$$

To project to vanishing total momentum, we sum over the spatial index $x$, a step which we suppress in the list of Eq. (2.3) for notational convenience. The interpolators are built from field variables at different lattice sites, and the integer valued parameters $n$ and $m$ determine how many steps in spatial direction the field variables are displaced.

Furthermore it is possible to have a relative minus sign between the displaced fields which gives rise to a derivative type of fermion source. In order to have the same quantum numbers as the combination with a plus sign, an additional factor of $\gamma_{1}$ appears. In 2-d all products of $\gamma$-matrices can be simplified to a single $\gamma_{\mu}$, or $\mathbb{1}$ due to the algebra of the Pauli matrices.

Our interpolators have negative parity, i.e., they acquire a minus sign under the parity transformation

$$
\psi(x, t) \stackrel{\mathbf{P}}{\longrightarrow} \psi(x, t)^{\mathbf{P}}=\gamma_{2} \psi(-x, t), \bar{\psi}(x, t) \stackrel{\mathbf{P}}{\longrightarrow} \bar{\psi}(x, t)^{\mathbf{P}}=\bar{\psi}(-x, t) \gamma_{2} .
$$

Furthermore all interpolators are eigenstates with $\mathbf{C}=-1$ of the charge conjugation

$$
\psi(x, t) \stackrel{\mathbf{C}}{\longrightarrow} \psi(x, t)^{\mathbf{C}}=C^{-1} \bar{\psi}(x, t)^{T}, \bar{\psi}(x, t) \stackrel{\mathbf{C}}{\longrightarrow} \bar{\psi}(x, t)^{\mathbf{C}}=-\psi(x, t)^{T} C,
$$

where $C$ is the charge conjugation matrix obeying $C \gamma_{\mu} C^{-1}=-\gamma_{\mu}^{T}$, which can be chosen as $C=i \gamma_{2}$. We stress that in 2-d no angular momentum exists and thus $P$ and $C$ completely characterize our flavor singlet states.

In our analysis we use two different choices for the displacement parameters $n$ and $m$. We set $n=3$ and $m=3$ to obtain the 9 interpolators $O_{1} \ldots O_{10}$ as listed in (2.3) (note that there is no interpolator $O_{7}$ ). A second choice, $n=4$ and $m=2$, gives rise to our interpolators $O_{11} \ldots O_{20}$ labeled accordingly. Thus we can use correlation matrices with a maximum size of $16 \times 16$.

The implementation of the correlation matrix was checked in two independent programs and compared to the results for the free case computed from Fourier transformation. Within error bars, the correlation matrix was found to be hermitian as expected. The decay properties of the individual entries in the correlation matrix are either of the cosh- or sinh-type. 

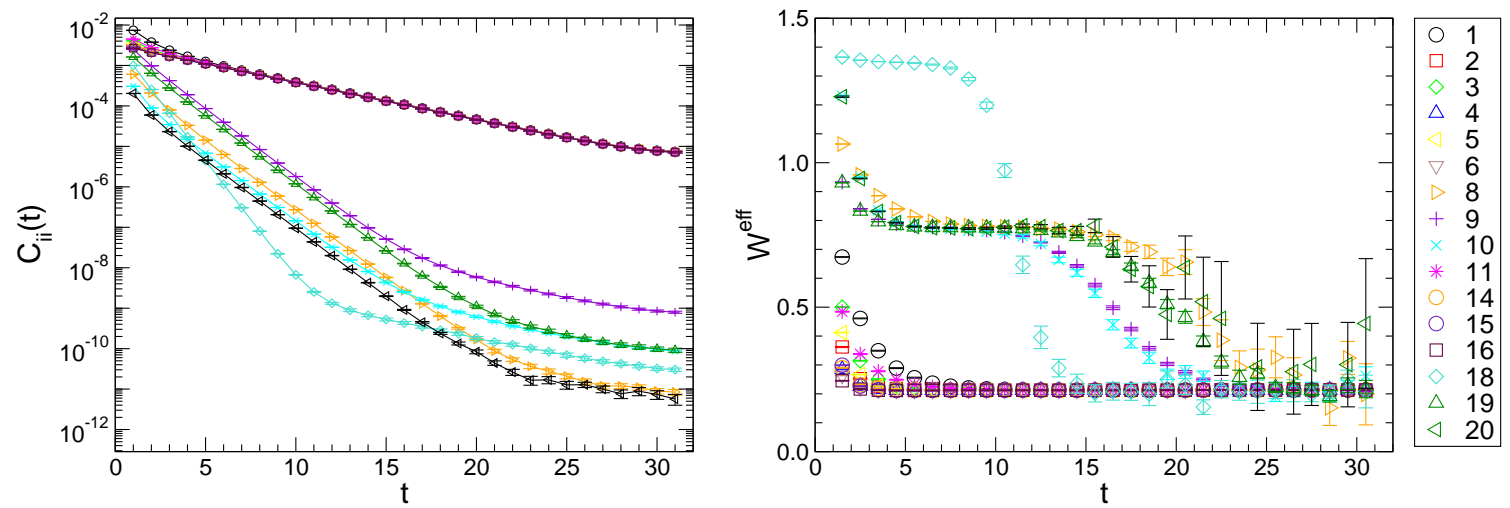

Figure 1: The diagonal entries $C_{i i}(t)$ of the correlation matrix (1.h.s. plot) and the corresponding effective energies (r.h.s) for a $16 \times 64$ lattice with $g=0.05, m=0.05$ and $N_{f}=2$. The numbers in the legend are the labels of our interpolators.

\section{Results}

For a first assessment of the data we consider plots of the correlators and of effective energies, a term which is more suitable than the usual "effective masses", since some of our states will turn out to be scattering states, such that their energy does not correspond to a single mass. The effective energies are defined as

$$
W^{\mathrm{eff}}(t+1 / 2)=\ln \frac{c(t)}{c(t+1)},
$$

where $c(t)$ is either an individual entry of the correlation matrix, or one of the eigenvalues of the generalized eigenvalue problem (1.2).

In the 1.h.s. plot of Fig. 1 we show the diagonal entries $C_{i i}(t)$ of the correlation matrix on a logarithmic scale. The r.h.s. plot contains the corresponding effective energies. The data are from $16 \times 64$ lattices at $g=0.05, m=0.05$ and $N_{f}=2$.

It is obvious from the 1.h.s. plot that on the logarithmic scale the correlators $C_{i i}(t)$ fall on a straight line beyond $t \sim 3$, i.e., are dominated by a single exponential. It is, however, very remarkable, that up to $t \sim 20$ they have rather different slopes, and only beyond that value they all settle for the smallest slope. In particular the interpolators with a relative minus sign (derivative interpolators) show a steeper slope compared to the interpolators with relative plus signs, indicating that the former strongly couple to excitations. We stress, however, that also the derivative interpolators couple to the ground state, as can be seen in the effective energy plots (r.h.s.): They clearly show a second plateau at the ground state energy for large values of $t$. Interpolator $O_{18}$ even seems to couple to a higher excited state, but settles at the ground state energy beyond $t \approx 13$. The fact, that some of our interpolators couple very strongly to excitations shows that they have a large overlap with the true physical states. It must, however, be understood, that the situation in 2-d is particularly simple due to the lack of angular momentum. All a spatial wave function can do is to have nodes in the single spatial direction. Such wave functions are easily obtained by linear combinations of our interpolators. 

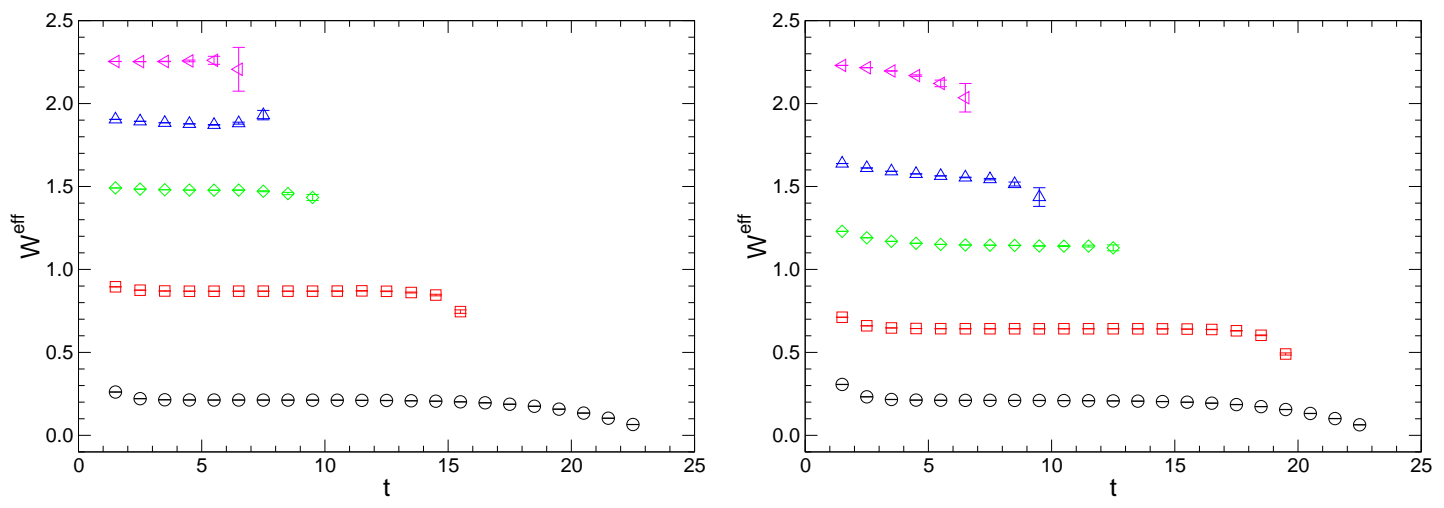

Figure 2: Effective energies from the eigenvalues of a $5 \times 5$ correlation matrix. We compare results from a $14 \times 48$ lattice (1.h.s.) to the data from $20 \times 48$ (r.h.s.), both for $g=0.05, m=0.05$ and $N_{f}=2$.

Let us now come to the analysis of the results from the variational analysis. In Fig. 2 we show the effective energies for the eigenvalues from the generalized eigenvalue problem for a $5 \times 5$ correlation matrix built from the interpolators $O_{3}, O_{5}, O_{8}, O_{18}$ and $O_{19}$ for $g=0.05, m=0.05$, $N_{f}=2$. We compare two different volumes, $14 \times 48$ in the 1.h.s. plot and $20 \times 48$ on the r.h.s.

The plots show long reliable plateaus, in particular for the three lowest states. The two highest states on the larger lattice (r.h.s.) show noticeable deviations from a horizontal line, which might be an effect of mixing with other states through the correction term in (1.3).

It is interesting to note that except for the lowest lying state (circles) all states show a pronounced volume dependence and are shifted towards smaller energies for the larger volume. This indicates that the higher states might be scattering states, which show, as discussed, a pronounced volume dependence. In a future contribution a more detailed analysis of the volume effects will be presented, which establishes that indeed the excited states of Fig. 2 are scattering states.

Here we would like to focus on a different aspect: For the finite volume analysis it is important to compare the energies from several different lattice volumes. However, when changing the volume, the spectrum is shifted and it is a non-trivial task to identify the individual states, in particular since two states might have changed their relative position. For the purpose of matching states on different volumes, the eigenvectors of the generalized eigenvalue problem (1.2) are an important tool. Since they contain the information on how a state is composed from the individual interpolators, they serve as a "fingerprint" for an individual state.

In Fig. 3 we display the entries of the eigenvectors (normalized to 1) for the ground state (1.h.s.) and our highest excited state (r.h.s.) as a function of $t\left(16 \times 48, g=0.01, m=0.05, N_{f}=2\right)$. Also the eigenvector entries form pronounced plateaus and the position of the plateau gives the coefficient for the corresponding interpolator in the linear combination which builds up the states. These coefficients are surprisingly stable for different volumes and thus are suitable quantities to be used in the identification of the states. Furthermore, the coefficients provide physically relevant information on how the states are composed: The ground state (1.h.s.) is dominated by the operators $\mathrm{O}_{3}$ and $\mathrm{O}_{5}$, whereas the highest excited state (r.h.s.) receives important contributions also from the derivative interpolators. Such an analysis allows for a qualitative understanding of the nature of the 

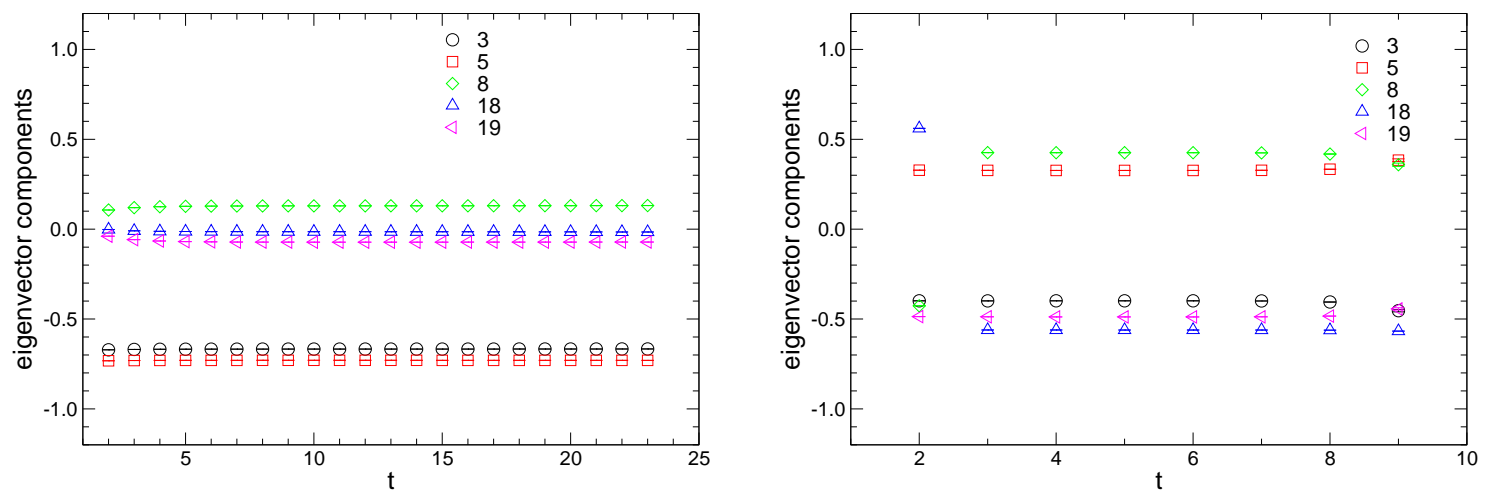

Figure 3: Entries of the eigenvectors for the ground state (1.h.s) and the fourth excitation (r.h.s) as a function of $t\left(16 \times 48, g=0.01, m=0.05, N_{f}=2\right)$. The numbers in the legend are labels of the interpolators.

excited states. In an upcoming paper we will use the methods presented here for a detailed analysis of the spectrum of excitations in the lattice Gross Neveu model.

\section{References}

[1] C. McNeile, PoS(LATTICE 2007)019 [arXiv:0710.0985 [hep-lat]]; T. Burch et al, Phys. Rev. D 74 (2006) 014504; Phys. Rev. D 73 (2006) 094505; Phys. Rev. D 73 (2006) 017502; Phys. Rev. D 70 (2004) 054502; S. Basak et al., arXiv:0709.0008 [hep-lat]; Phys. Rev. D 72 (2005) 074501; Phys. Rev. D 72 (2005) 094506; D. Brömmel et al Phys. Rev. D 69 (2004) 094513; S. Sasaki, T. Blum and S. Ohta, Phys. Rev. D65 (2002) 074503; K. Sasaki, S. Sasaki and T. Hatsuda, Phys. Lett. B 623 (2005) 208; J. M. Zanotti et al., Phys. Rev. D 68 (2003) 054506; W. Melnitchouk et al., Phys. Rev. D 67 (2003) 114506; N. Mathur et al., Phys. Lett. B 605 (2005) 137; F. X. Lee et al., Nucl. Phys. Proc. Suppl. 119 (2003) 296; S. Sasaki, Prog. Theor. Phys. Suppl. 151 (2003) 143.

[2] G. Fleming PoS(LATTICE2007)096; G.M. von Hippel, R. Lewis and R.G. Petry, PoS(LATTICE 2007)043 [arXiv:0710.0014 [hep-lat]]; D. Guadagnoli, M. Papinutto and S. Simula, Phys. Lett. B 604 (2004) 74; G.P. Lepage, Nucl. Phys. (Proc. Suppl.) 106 (2002) 12; M. Asakawa, T. Hatsuda and Y. Nakahara, Prog. Part. Nucl. Phys. 46 (2001) 459.

[3] C. Michael, Nucl. Phys. B259 (1985) 58.

[4] M. Lüscher and U. Wolff, Nucl. Phys. B339 (1990) 222.

[5] M. Lüscher, Nucl. Phys. B 364 (1991) 237; Nucl. Phys. B 354 (1991) 531.

[6] W. Bietenholz, S. Shcheredin and J. Volkholz, arXiv:0710.0997 [hep-lat]; U. Wolff, arXiv:0707.2872 [hep-lat]; C. Gattringer, V. Hermann and M. Limmer, Phys. Rev. D 76 (2007) 014503; C. Gattringer and I. Hip, Phys. Lett. B 480 (2000) 112; C.B. Lang and T.K. Pany, Nucl. Phys. B 513 (1998) 645; C. Gattringer, I. Hip and C.B. Lang, Phys. Lett. B 409 (1997) 371; C. Gattringer and C.B. Lang, Nucl. Phys. B 391 (1993) 463; W. Bietenholz, F. Hofheinz and J. Nishimura, JHEP 0209 (2002) 009; W. Bietenholz, A. Pochinsky and U.J. Wiese, Phys. Rev. Lett. 75 (1995) 4524.

[7] D.J. Gross and A. Neveu, Phys. Rev. D10 (1974) 3235. 